\title{
The Relationship Between Fetal and Maternal Hemodynamic Oscillations in Normal and Growth Restricted Fetuses
}

\author{
By Igor Lakhno*
}

\begin{abstract}
The study was aimed to the investigation of the maternal heart rate variability involvement in regulation of fetal circulatory system in case of healthy pregnancy and in fetal growth restriction. 98 patients at 34-40 weeks of gestation were enrolled in the study. And 30 of them were involved in Group I (control). In Group II 34 pregnant women with fetal growth restriction and normal Doppler indices were observed. 34 patients with decreased values of hemodynamic indices were monitored in Group III. The circulatory abnormalities in the utero-placental bed in patients with fetal growth restriction induced maternal and fetal autonomic response. The development of fetal compromise was due to an increased sympathetic and decreased parasympathetic tones. The fact of the considerable relationship between fetal and maternal heart rate variability in healthy pregnancy demonstrated the oscillatory nature of their interaction. Placental bed pathology in patients with fetal growth restriction and abnormal circulatory indices contributed to failed hemodynamic fluctuations exchange between mother and fetus.
\end{abstract}

Keywords: Fetal growth restriction, Fetal well-being, Heart rate variability, Hemodynamic fluctuations

\section{Introduction}

Fetal growth and maturation proceed in symbiotic relationship with maternal organism. Since fetal and maternal circulatory systems are anatomically separated the question of their relationship becomes rather relevant. It is possible to speculate the oscillatory origin of the interaction. Feto-placental hemodynamics is being determined by interaction of two main oscillators producing fluctuations, - maternal and fetal hearts (Van Leeuwen et al. 2009a). This cooperation depends on autonomic nervous regulation and follows the nonlinearity in the theory of continuous fluctuations or synergetics (Ivanov et al. 2009). The nonlinearity is provided by autonomic impact on the sinus node. Heart rate variability (HRV) is known as a convenient mechanism depending on the requirements of the oscillatory system (David et al. 2007, Di Pietro et al. 2010, May et al. 2014).

Feto-maternal interaction is associated with periods of cardiac synchrony. It was explored that maternal respiration provided the synchronization (Van Leeuwen et al. 2009b). Umbilical vein could be considered as a "mirror" of the oscillations in the system of "mother-placenta-fetus" since cord is not an innervated-tissue. Therefore, the variability of the blood flow velocities in the umbilical vein is determined by several external pace-makers of maternal

\footnotetext{
* Associate Professor of Perinatology, Obstetrics and Gynecology Department, Kharkiv Medical Academy of Postgraduate Education, Ukraine.
} 
origin. Fetal hemodynamic fluctuations captured maternal controlling signals (Di Pietro et al. 2010).

Fetal growth restriction (FGR) is a result of the chronic placental insufficiency (Aziz et al. 2012, Graatsma et al. 2012, Nassr et al. 2016, Vinkesteijn et al. 2004). Placental bed pathology deteriorates fetal hemodynamics. The lack of the placental circulation could decrease the penetration of the hemodynamic fluctuations through placental barrier. It is rather expedient to investigate the possible involvement of maternal HRV in the fetal circulatory response to FGR.

The aim of the investigation was to determine the role of maternal HRV in regulation of fetal circulatory system in case of healthy pregnancy and in FGR.

\section{Methodology}

The study protocol was approved by the Bioethics Committee of the Kharkiv Medical Academy of Postgraduate Education. The eligible participants were informed about the methodology of the study, its aims, objectives, indications and eventual complications before inclusion in the study. All patients who met the inclusion criteria gave written informed consent to participate in the investigation. The cases of the idiopathic FGR were determined. Inclusion criteria: FGR according to ultrasonography (Aziz et al. 2012). Exclusion criteria: multiple pregnancy, preexisting medical disorders like diabetes mellitus, metabolic syndrome, cardiac deseases, renal desease, thyrotoxicosis. The patients with pregestational (chronic hypertension, diabetic vasculopathy) systemic vascular pathologies were excluded from the study. The study was conducted from January 2014 to October 2015.

98 patients at 34-40 weeks of gestation were enrolled in the study. And 30 of them had healthy pregnancy and were involved in Group I (control). In Group II 34 pregnant women with FGR and normal Doppler ultrasonographic indices were observed. 34 patients with decreased values of utero-placental or feto-placental hemodynamics determined by hemodynamic Doppler were monitored in Group III.

Doppler ultrasonography was performed on the ultrasound system "Voluson 730" (GE Healthcare, USA). The obtained within 1 minute Doppler spectrogram of the venous umbilical blood flow was subjected to further processing. The curves of maximum blood flow velocity were isolated and their spectral components were determined. The spectra were calculated by sampling step $\Delta t=0.01$ seconds for a sample of 256 points. The resulting spectrum was obtained by averaging over all samples of this contingent.

Fetal and maternal HRV parameters were obtained with the application of fetal non-invasive computer electrocardiographic system "Cardiolab Baby Card" (Scientific Research Center "KhAI-Medica", Ukraine). The ukrainian ECG recordings were included in Physio Net database (Silva et al. 2010). The registration lasted for 10 minutes in the maternal sitting position. The values of total power (TP) and its spectral compounds: very low frequency (VLF), low 
frequency (LF) and high frequency (HF) were determined. The temporal characteristics of the fetal HRV: standard deviation of normal to normal intervals (SDNN), root mean square of successive heartbeat interval differences (RMSSD), the proportion of the number of pairs of NNs that differ by more than $50 \mathrm{~ms}$ divided by total number of NNs (pNN50), the amplitude of mode (the most frequent value of $\mathrm{NN}$ interval or the highest column in the histogramm) - number of $\mathrm{NN}$ intervals included in the pocket corresponding to mode measured in percents (\%) (AMo) and stress index $\mathrm{SI}=\mathrm{AMo}(\%) /(2 \times \mathrm{Mo} \times \mathrm{Var}) ; \mathrm{Var}=\mathrm{NNmax}-\mathrm{NNmin} ;(\mathrm{SI})$ were calculated.

The results thus obtained were analyzed using the ANOVA test to compare for categorical data between the Groups. The significance was set at $\mathrm{p}$-value $<0.05$. For the statistical analysis of relationship between $\mathrm{X}$ and $\mathrm{Y}$, correlations coefficients were estimated using Spearman's test. Microsoft Office 2010 Excel software was used for statistical analysis (Washington, USA).

\section{Results}

Table 1. Maternal HRV Parameters in the Study Population

\begin{tabular}{|l|c|c|c|}
\hline Index & Group I & Group II & Group III \\
\hline SDNN, ms & $118.9 \pm 16.4$ & $118.1 \pm 18.3$ & $114.2 \pm 19.5 * / \uparrow$ \\
\hline RMSSD, ms & $41.5 \pm 7.3$ & $41.0 \pm 8.4$ & $40.6 \pm 9.1$ \\
\hline pNN5O, \% & $12.7 \pm 2.5$ & $12.6 \pm 3.1$ & $12.4 \pm 3.1$ \\
\hline AMo, \% & $34.5 \pm 6.0$ & $34.6 \pm 6.8$ & $40.8 \pm 8.3 * / \uparrow$ \\
\hline SI, c.u. & $115.2 \pm 16.6$ & $117.0 \pm 20.1$ & $180.7 \pm 31.2 * / \uparrow$ \\
\hline TP, ms ${ }^{2}$ & $3091.4 \pm 421.8$ & $3087.9 \pm 409.7$ & $2889.0 \pm 334.2 * / \uparrow$ \\
\hline VLF, ms ${ }^{2}$ & $2370.1 \pm 346.7$ & $2368.4 \pm 181.4$ & $2367.6 \pm 165.6$ \\
\hline LF, ms ${ }^{2}$ & $343.0 \pm 47.4$ & $342.9 \pm 52.4$ & $286.3 \pm 41.9 * / \uparrow$ \\
\hline HF, ms ${ }^{2}$ & $378.3 \pm 53.5$ & $377.1 \pm 56.2 *$ & $236.5 \pm 39.6 * / \uparrow$ \\
\hline LF/HF & $0.9 \pm 0.2$ & $0.9 \pm 0.3$ & $1.2 \pm 0.4 * \uparrow$ \\
\hline
\end{tabular}

Notes: $*$ - the differences were statistically significant compared to Group I $(\mathrm{p}<0.05)$;

$\dagger$ - the differences were statistically significant compared to Group II $(\mathrm{p}<0.05)$.

Abbreviations: SDNN - the standard deviation of normal to normal intervals; RMSSD - the root mean square of successive heartbeat interval differences; pNN50 - the proportion of NN pairs differing by more than $50 \mathrm{~ms}$ divided by total number of NNs; AMo - the mode amplitude (the most frequent value of NN interval or the highest column in the histogramm) the number of $\mathrm{NN}$ intervals included into the pocket corresponding to the mode measured in percentages (\%) (AMo); SI - the stress index SI=AMo $(\%) /(2 \times M o \times V a r)$; Var=NNmaxNNmin; TP - the total power; VLF - the very low frequency; LF - the low frequency; HF the high frequency.

The investigation of maternal HRV revealed almost equal autonomic tone in the study population (Table 1). The most considerable power was determined in the VLF domain region in all study Groups. It was due to a relative predominance of the hypothalamic-pituitary-adrenal axis domain region power among all spectral components of HRV. The maternal HRV in Group III demonstrated an increased sympathetic activity. It was found the 
relative increase of AMo, SI and LF. Sympathetic overactivity could be speculated as an adaptive response to the abnormal utero-placental hemodynamics. So, this regulatory shift captured adrenergic compensatory reaction focused on fetal trophotropic support. The mean sympatho-vagal balance (LF-to-HF ratio) values were $0.9 \pm 0.2,0.9 \pm 0.3$ and $1.2 \pm 0.4$ respectively in Group I, Group II and Group III. The mean values of nonlinear short-term parameters: RMSSD, pNN5O were same in all Groups. Therefore, maternal autonomic tone stayed constant in case of FGR without hemodynamic disturbances. The explored slight increase of sympathetic activity reduced the level of autonomic regulation in Group III.

The fetal HRV parameters demonstrated a decreased autonomic nervous regulation with a relative elevation of the sympathetic domain region values in Group III (Table 2). The fetal autonomic tone and the power of its spectral domain regions in case of FRG without hemodynamic disturbances were almost equal to healthy pregnancy Group. The values of SDNN and TP were lower in Group III. However, the values of AMo, SI and LF were increased in FGR with abnormal hemodynamic indices. The decrease of RMSSD, pNN5O and HF was determined in Group III. So, a lack of the fetal vagal division of autonomic nervous regulation in FGR with hemodynamic disturbances was found. The explored decline of VLF values in Group III could reflect the decreased level of ergo-, trophotropic processes. Therefore, FGR is associated with fetal autonomic imbalance in case of utero-placental or umbilical hemodynamics failure.

Table 2. Fetal HRV Parameters in the Study Population

\begin{tabular}{|l|c|c|c|}
\hline Index & Group I & Group II & Group III \\
\hline SDNN, ms & $45.6 \pm 10.4$ & $44.9 \pm 9.6$ & $36.2 \pm 8.5 * / \uparrow$ \\
\hline RMSSD, ms & $22.5 \pm 6.0$ & $22.3 \pm 6.4$ & $16.7 \pm 4.2 * / \uparrow$ \\
\hline pNN5O, \% & $4.2 \pm 0.8$ & $4.1 \pm 1.0$ & $3.2 \pm 0.6 * / \uparrow$ \\
\hline AMo, \% & $38.9 \pm 7.4$ & $39.1 \pm 8.5$ & $51.3 \pm 10.3 * / \uparrow$ \\
\hline SI, c.u. & $160.2 \pm 25.6$ & $162.7 \pm 31.4$ & $583.6 \pm 96.9 * / \uparrow$ \\
\hline TP, ms ${ }^{2}$ & $1501.4 \pm 247.8$ & $1504.8 \pm 229.3$ & $973.5 \pm 187.2 * / \uparrow$ \\
\hline VLF, ms ${ }^{2}$ & $1255.8 \pm 196.3$ & $1257.0 \pm 189.6$ & $669.4 \pm 79.1 * / \uparrow$ \\
\hline LF, $\mathrm{ms}^{2}$ & $170.1 \pm 35.5$ & $169.2 \pm 33.9$ & $241.5 \pm 48.2 * / \uparrow$ \\
\hline HF, $\mathrm{ms}^{2}$ & $76.9 \pm 10.3$ & $78.1 \pm 9.3$ & $63.0 \pm 12.6 * / \uparrow$ \\
\hline
\end{tabular}

Notes: $\quad *$ - the differences were statistically significant compared to Group I $(\mathrm{p}<0.05)$;

$\dagger-$ the differences were statistically significant compared to Group II $(\mathrm{p}<0.05)$.

Abbreviations: SDNN - the standard deviation of normal to normal intervals; RMSSD - the root mean square of successive heartbeat interval differences; pNN50 - the proportion of NN pairs differing by more than $50 \mathrm{~ms}$ divided by the total number of NNs; AMo - the mode amplitude (the most frequent value of NN interval or the highest column in the histogramm) the number of NN intervals included in the pocket corresponding to mode measured in percentages $(\%)$; SI - the stress index $\mathrm{SI}=\mathrm{AMo}(\%) /(2 \times \mathrm{Mo} \times \mathrm{Var})$; Var=NNmax-NNmin; TP the total power; VLF - the very low frequency; LF - the low frequency; HF - the high frequency.

The obtained spectral characteristics of the venous blood flow velocity revealed the possible pace-makers in the study population. The hemodynamics 
in the umbilical vein was characterized with three mostly pronounced spectral peaks: $0.5 \mathrm{~Hz}, 2 \mathrm{~Hz}$ and $7 \mathrm{~Hz}$ (Figure 1). The amplitudes of the abovementioned peaks in healthy pregnancy Group and Group II were equal (Table 3 ). It was explored the elevation of $2 \mathrm{~Hz}$-associated peak and the decrease of the $0.5 \mathrm{~Hz}$ - and $7 \mathrm{~Hz}$-mediated peaks in FGR with hemodynamic disturbances Group. It was found an evident pulsation of the venous wall in Group III.

Figure 1. The Spectral Characteristics of the Umbilical Venous Blood Flow Velocity in Group I

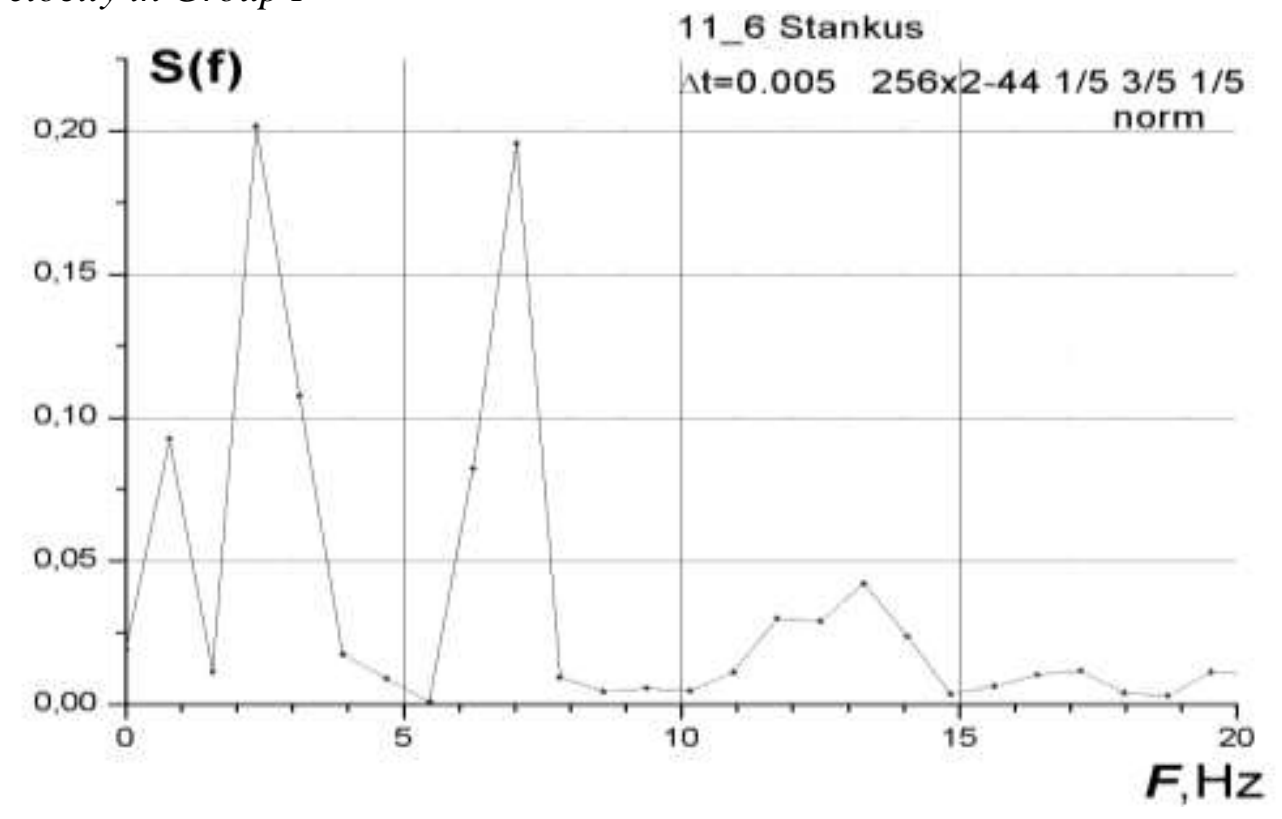

Table 3. The Amplitudes of the Spectral Peaks of Umbilical Blood Flow Velocity in the Study Population

\begin{tabular}{|l|c|c|c|}
\hline $\begin{array}{l}\text { Frequency of } \\
\text { peak }\end{array}$ & Group I & Group II & Group III \\
\hline $0,5 \mathrm{~Hz}$, c.u. & $0.19 \pm 0.04$ & $0.18 \pm 0.03$ & $0.08 \pm 0.02 * / \uparrow$ \\
\hline $2 \mathrm{~Hz}$, c.u. & $0.16 \pm 0.03$ & $0.16 \pm 0.05$ & $0.18 \pm 0.04 * / \uparrow$ \\
\hline $7 \mathrm{~Hz}$, c.u. & $0.18 \pm 0.03$ & $0.18 \pm 0.04$ & $0.06 \pm 0.02 * / \uparrow$ \\
\hline
\end{tabular}

Notes: $\quad *-$ the differences were statistically significant compared to Group I $(\mathrm{p}<0.05)$;

$\dagger$-the differences were statistically significant compared to Group II ( $\mathrm{p}<0.05)$.

The investigation of statistically significant correlations between the maternal and fetal SDNN values and the amplitude of $0.5 \mathrm{~Hz}$-associated peak in the study population revealed certain regularities (Table 4). The most considerable positive correlation was determined in healthy pregnancy Group between the maternal SDNN and the $0.5 \mathrm{~Hz}$-associated peak amplitude $(\mathrm{R}=0.68 ; \mathrm{p}<0.05)$, the maternal and the fetal SDNNs $(\mathrm{R}=0.46 ; \mathrm{p}<0.05)$. The force of correlation was identical to Group I in the same pairs in Group II. The weak correlation between the maternal SDNN and the amplitude of $0.5 \mathrm{~Hz}$ frequency peak $(\mathrm{R}=0.20 ; \mathrm{p}<0.05)$ was explored in Group III. No relationship was revealed between the maternal and the fetal SDNNs $(\mathrm{R}=0.12 ; \mathrm{p}<0.05)$ in 
Group III. Therefore, hemodynamic disturbances in fetoplacental system decreased the force of correlation between fetal and maternal autonomic tone.

Table 4. Statistically Significant $(P<0.05)$ Spearman's Correlations Between the Maternal SDNN and the Amplitude of $0.5 \mathrm{~Hz}$-Associated Peak, the Maternal and Fetal Sdnns in the Study Population

\begin{tabular}{|c|c|c|c|}
\hline Pairs of parameters (X versus Y) & Group I & Group IIGroup III \\
\hline $\begin{array}{c}\text { Amplitude of the } 0.5 \mathrm{~Hz} \text {-associated peak vs } \\
\text { maternal SDNN }\end{array}$ & $\mathrm{R}=0.68$ & $\mathrm{R}=0.66$ & $\mathrm{R}=0.20$ \\
\hline Maternal SDNN vs fetal SDNN & $\mathrm{R}=0.46$ & $\mathrm{R}=0.42$ & $\mathrm{R}=0.12$ \\
\hline
\end{tabular}

\section{Discussion}

The typical pattern of autonomic power spectrum is associated with an increased activity of vagal-mediated regulation in the I half of healthy pregnancy (Schobel et al. 1996). The above-mentioned gestational resetting is involved in the mechanism of fluid retention. And it also provides decreased vascular tone in the process of systemic vasodilation (Yang et al. 2000, Musa et al. 2016). The placental circuit of hemodynamics becomes a zone of the high vascular resistance in case of chronic placental insufficiency (Arias-Ortega et al. 2015, Aziz et al. 2012, Tachibana et al. 2016). The circulatory abnormalities in the utero-placental bed in patients with FGR induced maternal and fetal autonomic response. The revealed maternal sympathetic overactivity was found as an adaptive reaction aimed to an improved feto-maternal hemodynamics. FRG without hemodynamic disturbances featured by normal autonomic pattern of regulation. The development of fetal compromise in case of the abnormal utero-placental or umbilical circulation was due to an increased sympathetic and decreased parasympathetic tones.

Umbilical venous hemodynamics had three evident pace-makers. The controlling signal with a frequency about $2 \mathrm{~Hz}$ was associated with fetal cardiac activity and arterial component of the circulation. The pulsatile pattern of the venous umbilical hemodynamics is known as an evident marker of fetal distress (Vinkesteijn et al. 2004). Two other pace-makers revealed in the umbilical vein took part in the adjustment of continuous non-pulsatile type of blood flow. These drivers had an external origin and provided fetal nutrition. The $0.5 \mathrm{~Hz}$-associated pace-maker was possibly driven by maternal hemodynamic fluctuations or HRV. The explored correlation in pair the amplitude of $0.5 \mathrm{~Hz}$-associated peak versus maternal SDNN confirmed this speculation. The fact of the considerable relationship between fetal and maternal HRV in healthy pregnancy demonstrated the oscillatory nature of their interaction. Placental bed pathology in patients with FGH and abnormal circulatory indices contributed to failed hemodynamic fluctuations exchange between mother and fetus. The relation between maternal and fetal organisms almost disappeared in FGR with hemodynamic disturbances. The nature of 7 $\mathrm{Hz}$-associated driver is unclear. This frequency is known to be driven by natural Earth magnetic field, brain bioelectrical activity or DNA waves 
(Pitkanen 2011). The above-mentioned fields are very weak and could hardly produce evident hemodynamic fluctuations.

This investigation demonstrated a novel approach to the evaluation of fetal well-being. Since chronic placental insufficiency is known as a preliminary stage of pre-eclampsia the revealed values of the hemodynamic fluctuations could be used for the prediction of pre-eclampsia.

\section{Conclusions}

HRV or hemodynamic fluctuations were an evident mechanism of fetal and maternal hemodynamic coupling. This interaction captured maternal HRV repercussions on fetal autonomic nervous regulation and hemodynamics. The autonomic repercussions theory postulated that fluctuations propagated to the fetus through placental barrier. The speculation that maternal HRV is a driver of the fetal umbilical venous hemodynamics was supported. Therefore, maternal autonomic tone provided fetal nutrition in healthy pregnancy. HRV was determined as a convenient instrument of the regulation of hemodynamics in the system of "mother-placenta-fetus". Placental bed pathology in FGR deteriorated propagation of the fluctuations and destroyed maternal and fetal interaction. The increased power of maternal sympathetic domain region was revealed as a compensatory reaction due to deteriorated utero-placental hemodynamics. Sympathetic overactivity modulated maternal autonomic response by reducing $\mathrm{TP}$ and suppressing parasympathetic regulation. The investigation of the spectral compounds of the umbilical venous circulation confirmed the maternal origin of $0.5 \mathrm{~Hz}$ peak. The value of the amplitude of $0.5 \mathrm{~Hz}$ peak could be considered as a biophysical marker of FGR and preeclampsia. As pre-eclampsia is a result of the chronic placental insufficiency the decreased amplitude of the above-mentioned peak, maternal and fetal autonomic tone could be speculated as an additional criteria of such gestational disease. The screening for pre-eclampsia in III trimester of pregnancy is necessary to determine indications for the preterm pregnancy termination. The proposed criteria of PE could be investigated in future as markers of its severity. Maternal sympathetic overactivity requires further investigations in case of healthy pregnancy and in pathological conditions. Hemodynamic regimen in the fetoplacental system possibly captures the autonomic disorders in latent and clinically manifested forms of pre-eclampsia. The postulated approach to the diagnosing of pre-eclampsia is an evident step in the clinical introduction of the theory of non-linearity or synergetics. The further investigations of the role of cardiovascular oscillations could promote to the advancement and evolution of gestational pathology. 


\section{References}

Arias-Ortega R, Echeverria JC, Gusman-Huerta M, Camargo-Marín L, GaitánGonzález MJ, Borboa-Olivares H et al. (2015) Respiratory sinus arrhythmia in growth restricted fetuses with normal Doppler hemodynamic indices. Early Human Development 93: 17-26.

Aziz W, Schlindwein FS, Wailoo M, Biala T, Rocha FC (2012) Heart rate variability analysis of normal and growth restricted children. Clinical Autonomic Research 22(2): 91-97.

David M, Hirsch M, Karin J, Toledo E, Akselrod S (2007) An estimate of fetal autonomic state by time-frequency analysis of fetal heart rate variability. Journal of Applied Physiology 102(3): 1057-1064.

DiPietro J, Kivlighan K, Costigan K, Rubin SE, Shiffler DE, Henderson JL et al. (2010) Prenatal antecedents of newborn neurological maturation. Child Development 81:115-130.

Graatsma EM, Mulder EJH, Vasak B, Lobmaier SM, Pildner von Steinburg S, Schneider KT et al. (2012) Average acceleration and deceleration capacity of the fetal heart rate in normal pregnancy and in pregnancies complicated by fetal growth restriction. The Journal of Maternal- Fetal and Neonatal Medicine 25(12): 2517-2522.

Ivanov PC, Qianli DYM, Bartsch RP (2009) Maternal-fetal heartbeat phase synchronization. PNAS-Proceedings of the National Academy of Sciences 106(33): 13641-13642.

May LE, Scholtz SA, Suminski R, Gustafson KM (2014) Aerobic exercise during pregnancy influences infant heart rate variability at one month of age. Early Human Development 90(1): 33-38.

Musa SM, Adam I, Lutfi MF (2016) Heart Rate Variability and Autonomic Modulations in Preeclampsia. PLoS One 11(4): e0152704.

Nassr AA, Abdelmagied AM, Shazly SA (2016) Fetal cerebro-placental ratio and adverse perinatal outcome: systematic review and meta-analysis of the association and diagnostic performance. Journal of Perinatal Medicine 44(2): 249-256.

Pitkanen M (2011) DNA \& water memory: comments on Montagnier group's recent findings. DNA Decipher Journal 1(1): 181-190.

Silva I, Behar J, Sameni R, Zhu T, Oster J, Clifford GD et al. (2010) Noninvasive Fetal ECG: the PhysioNet/Computing in Cardiology Challenge 2013. Computing in Cardiology 40: 149-152.

Schobel HP, Fischer T, Heuszer K, Geiger H, Schmieder RE (1996) Preeclampsia - a state of sympathetic overactivity. New England Journal of Medicine 335: 1480-1485.

Tachibana M, Nakayama M, Miyoshi Y (2016) Placental examination: prognosis after delivery of the growth-restricted fetus. Current Opinion in Obstetrics and Gynecoly 28(2): 95-100.

Van Leeuwen P, Geue D, Lange S, Gronemeyer D (2009a) Modeling fetal-maternal heart-rate interaction. IEEE Engineering in Medicine and Biology Magazine 28(6): 49-53.

Van Leeuwen P, Geue D, Thiel M, Cysarzd D, Langeb S, Romanoc MC (2009b) Influence of paced maternal breathing on fetal-maternal heart rate coordination. PNAS-Proceedings of the National Academy of Sciences 106(33): 13661-13666.

Vinkesteijn AS, Struijk PC, Ursem NT, Hop WC, Wladimiroff JW (2004) Fetal heart rate and umbilical artery flow velocity variability in intrauterine growth 
restriction: a matched controlled study. Ultrasound in Obstetrics and Gynecology 23(5): 461-465.

Yang CCH, Chao T, Kuo BJK, Yin CS, Chen HI (2000) Preeclamptic pregnancy is associated with increased sympathetic and decreased parasympathetic control of HR. American Journal of Physiology - Heart and Circulatory Physiology 278(4): H1269-1273. 
